\title{
Science of Synthesis: Multicomponent Reactions, Workbench Edition, 2 Vol.
}

Edited by Thomas J. J. Müller, $1^{\text {st }}$ ed., Thieme: Stuttgart, 2014, 1278 pp, \$ 549, ISBN: 9783131765611

Multicomponent reactions have been providing scientists with very efficient strategies for the construction of complicated molecules with biological or medicinal potential. This two-volume reference book edited by Thomas J. J. Müller with a nice collection of contributions from the experts in this area contains the information of the recent advances on this specific topic. The first volume deals with carbonyl-based multiple reactions, while the second summarizes the reactions of $\alpha, \beta$-unsaturated carbonyl reactions, cycloaddition, and metal-mediated reactions. They start with well-designed abstracts containing a brief summary of each chapter followed by a graphical abstract and addition of keywords, telling the readers the main contents. In the first chapter, the volume editor discusses the relative reactivities of functional groups, which have been identified as the most important principle to design multicomponent reactions. The second chapter has been well organized for the reaction of carbonyl compounds as electrophilic components with amines or analogues as one nucleophilic component and a third component as the subtitle. Finally Ugi-type reactions of isocyanides with activated alkynes and Gewald reactions of carbonyl compounds, sulfur and nitriles have also been discussed. The second volume starts with a chapter on multicomponent reactions of $\alpha, \beta$-unsaturated electron-deficient alkenes and alkynes. The second chapter deals with the reactions involving cycloadditions. The third chapter is on boron-involved coupling reactions and the fourth on silicon compound mediated reactions. The fifth chapter is very attractive, summarizing the most important advances on radical reactions in the presence of carbon monoxide. Metal complexes have been found to be very useful for the construction of cyclic skeletons. The last two chapters are left for such advances with stoichiometric and catalytic amounts of metal species, respectively.

In conclusion, although there is some minor overlap in some chapters, it is well structured: easy for readers to get the most important knowledge in this area; one may also readily find the required specific information by just referring to the (sub)title and abstract. They are not only nice reading material for senior undergraduate or graduate students but also a set of very good reference books for the researchers working both in academic chemical laboratories and industries, especially those in drug discovery.

Prof. Dr. Shengming Ma, Professor at East China Normal University and Shanghai Institute of Organic Chemistry 